\title{
Actitudes hacia las matemáticas: un estudio en una escuela rural de la Costa Caribe Sur de Nicaragua
}

\author{
Eliel Jiménez Bonilla' \\ William Oswaldo Flores López²
}

\section{Resumen}

G esta investigación se han analizado las actitudes hacia las matemáticas del estudiantado de Educación ESecundaria procedentes de una escuela rural. Es un estudio cuantitativo sustentado en el diseño descriptivo donde se suministró una escala de actitud hacia las matemáticas a 6o estudiantes de Educación Secundaria Rural. Se realizó análisis descriptivo de los datos, una prueba t de student, análisis de varianza y prueba HSD-Tukey con el Paquete Estadístico para las Ciencias Sociales (SPSS). Los resultados muestran que: la actitud hacia las matemáticas es positiva; los factores agrado, utilidad, motivación y confianza se manifiestan contribuyendo a que la ansiedad hacia las matemáticas sea menor; hombres y mujeres tienen el mismo grado de actitud; y no existen diferencias estadísticamente significativas en relación al grado de estudio. Se concluye que las actitudes hacia las matemáticas son parte de la formación del estudiantado de Educación Secundaria Rural, ya que les permite desarrollar habilidades y destrezas para conocer, valorar, comprender, manejar las emociones y sentimientos de forma armónica en la resolución de situaciones matemáticas vinculadas con la vida cotidiana.

Palabras clave: Ansiedad; Agrado; Utilidad; Motivación; Confianza; Género.

\section{Abstract}

In this research, it has been analyzed the mathematical attitudes of High School students from a rural school. It is a quantitative study based on the descriptive design where a scale of attitudes towards mathematics was provided to 6o students of Rural High School. Descriptive analysis of the data, a t student's test, analysis of variance and HSD-Tukey test with the Statistical Package for the Social Sciences (SPSS) were performed. The results show that: the attitude toward mathematics is positive; factors liking, usefulness, motivation and confidence manifested, are contributing to demonstrate that math anxiety is less; Men and women have the same degree of attitude, and there are no statistically significant differences related to the degree of study. It is concluded that attitudes towards mathematics are part of students' training from rural high school, as it allows them to develop skills and abilities to know, value, understand, emotions and feelings management in a harmonious way in the resolution of mathematical situations related with everyday life.

Keywords: Anxiety; Liking; Utility; Motivation; Trust; Gender.

\footnotetext{
1 Licenciado en Ciencias de la Educación con Mención en Matemáticas, Asesor Pedagógico de Matemáticas, MINED. Correo: ejimenezbonilla@gmail.com

2 Doctor en Educación, Profesor Investigador de la Universidad de las Regiones Autónomas de la Costa Caribe Nicaragüense. Correo: william.flores@uraccan.edu.ni
}

Recibido: 05/03/2017 - Aprobado: 12/06/2017 


\section{Introducción}

La matemática es una de las asignaturas más relevantes en el Currículo de Educación Secundaria Rural, ya que toda persona que vive y se desarrolla en la comunidad rural, utiliza las matemáticas para resolver situaciones de la vida cotidiana, por tanto, es necesario que aprenda matemáticas de una manera formal, es decir, aprender a: pensar y razonar; argumentar y justificar; comunicar, modelar, representar; $y$, plantear y resolver problemas de las situaciones que día a día se le presenta en el mundo real. No obstante, las matemáticas se han constituido en un obstáculo en el estudiantado en su formación académica, manifestándose en deficiencias como un desconocimiento de los algoritmos, así como una carencia en el planteamiento y resolución de problemas.

Los estudios de Hidalgo, Maroto y Palacios (2005), revelan que cuando el estudiantado comienza su proceso de formación escolarizada en el primer grado, el 87 por ciento posee un alto interés ante la resolución de problemas matemáticos, pero a medida que avanzan en su trayecto formativo, dicho gusto por esta disciplina va disminuyendo de forma gradual, hasta llegar a un nivel secundario con un porcentaje del 50\% (Gil, Guerrero y Blanco, 2006) consideran que son muchos los estudiantes que generan en el transcurso de su vida académica actitudes negativas hacia las matemáticas, manifestando, en ocasiones, una auténtica aversión y/o rechazo hacia esta disciplina. Para una mayoría de los estudiantes, esta materia no es una fuente de satisfacción, sino de frustración, desánimo y angustia. A muchos de ellos, incluyendo a algunos de los más capacitados, les desagradan y agobian las matemáticas.

Por consiguiente, la presente investigación tiene como propósito analizar las actitudes hacia las matemáticas del estudiantado de Educación Secundaria procedente de una escuela rural, sabiendo que, un objetivo en la enseñanza y aprendizaje de las matemáticas es que el estudiantado desarrolle actitudes, creencias y emociones que aumenten sus probabilidades de utilizar con éxito las matemáticas.

\section{Revisión de la literatura}

Las actitudes son predisposiciones evaluativas de conducta que determinan las intenciones personales e influye en el comportamiento, y que consta de un componente cognitivo, un componente afectivo y un componente institucional (GómezChacón, 2000). Cuando se trata de actitudes matemáticas, se puede distinguir dos grandes categorías reconocidas también por la NCTM (2000): actitudes hacia las matemáticas y actitudes matemáticas. Las primeras se ven más afectadas por el componente afectivo, y las segundas, por lo cognitivo.

Algunas de las actitudes y comportamientos más habituales en el proceso de aprendizaje que manifiestan los estudiantes son el rechazo, la negación, la frustración y la evitación, por lo que se hace necesario el estudio de las mismas sobre todo si éstos continúan con su formación académica después de haber egresado de la Educación Primaria. Como lo menciona Schofield (1982), uno de los aspectos que más se correlaciona con la realización matemática es el agrado-temor que la persona siente hacia esta disciplina, presentándose los dos polos del elemento que comúnmente se denomina ansiedad hacia las matemáticas. Así, es importante desarrollar actitudes positivas en los estudiantes, lo cual facilitará un cambio en las creencias y expectativas hacia la materia, favoreciendo su acercamiento hacia las mismas por lo que es prioritario investigar desde el inicio de un ciclo formativo el tipo de actitudes que poseen los estudiantes.

Desde esta perspectiva, Auzmendi (1992) establece que los dominios que inciden en la actitud hacia las matemáticas son: agrado, ansiedad, utilidad, motivación y confianza, los cuales son constructos que son relevantes de analizar como elementos de diagnóstico que permitan obtener información relevante acerca de las creencias de los estudiantes antes de iniciar un curso. Del mismo modo, Lim, Tso y Lin (2009), concluyeron que las actitudes hacia esta disciplina es un fenómeno multidimensional, encontrando que estos componentes influyen en el aprendizaje de los estudiantes porque les ofrece un panorama de las interrelaciones de las matemáticas en la vida real. Como lo menciona Calvo y Susinos (2010) es relevante que los docentes promuevan en los educandos un saber vivo y participativo sobre la disciplina que aprenden, así como generar en el aula un espacio de relación en el que se presenten intercambios fructíferos, acogedores y estimulantes que les permitan vivir experiencias gratas para así generar actitudes positivas hacia las diversas materias de la escuela. 


\section{Materiales y métodos}

El enfoque de esta investigación es cuantitativo porque este paradigma ayudó al tratamiento de la información de los datos a través de la descripción de las propiedades, características y perfiles de las personas, grupos, comunidades, procesos y objetos o cualquier otro fenómeno que se someta a un análisis (Hernández, Fernández, \& Baptista, 2010). Mientras que el diseño que sitúa a esta investigación es descriptivo ya que tiene como objetivo indagar la incidencia de las modalidades o niveles de una o más población (Bisquerra, 2012).

El estudio se llevó a cabo con 60 hombres y mujeres estudiantes de Educación Secundaria de una escuela rural de la comunidad de Campana del Municipio Muelle de los Bueyes con edades comprendidas entre 11 y 20 años. El 53 por ciento de los participantes fueron mujeres y el 47 por ciento restante hombres. Para concluir, indicar que el tipo de muestreo que hemos utilizado ha sido el muestro no probabilístico causal o accidental, que es aquel en el cual el investigador selecciona directa e intencionalmente la muestra, debido fundamentalmente a que tiene fácil acceso a la misma y es representativa de la población (Gil, Rodríguez \& García, 1995; Albert, 2006; Sabariego, 2004).

El instrumento para la recopilación de la información fue el cuestionario de actitudes hacia las matemáticas de Auzmendi (1992). El instrumento está constituido por 25 ítems, aglutinados en 5 factores asociadas a las actitudes hacia las matemáticas. El estudiantado debía indicar su grado de acuerdo o desacuerdo con afirmaciones propuesta por medio de una escala de Likert de 5 valores. A continuación, se describen los 5 factores o dimensiones del instrumento:

- Agrado. Constituido por 4 ítems, este factor hace referencia al aspecto de agrado o disfrute que provoca el trabajo matemático.

- Ansiedad. Integrado por 9 ítems, este factor se refiere al sentimiento de ansiedad, temor que el estudiantado manifiesta ante la materia.

- Motivación. Compuesto por 3 ítems, este factor puede interpretarse como la motivación que siente el estudiantado hacia el estudio y utilización de las matemáticas.

- Utilidad. Configurada por 6 ítems, este factor hace referencia al valor que el estudiantado otorga a las matemáticas, a la utilidad que él estudiantado percibe que puede tener esta materia para su futura vida profesional.
- Confianza. Establecida por 3 ítems, este factor puede interpretarse como sentimiento de confianza que provoca la habilidad en matemáticas.

Se adaptó el instrumento para su aplicación en estudiantes de Educación Secundaria, principalmente, para estudiantes de escuelas rurales. Para ello se aplicó un estudio socio métrico al instrumento, para comprobar los valores de validez y fiabilidad del mismo. Se calculó el valor de consistencia interna del total de los ítems del cuestionario, obteniendo un valor alfa de Cronbach del 90 por ciento de confiabilidad. Con respecto a la validez, se realizó un análisis de componentes principales (ACP). La prueba Kaiser-Meyer-Olkin arrojo una puntuación de 0,907 . Por su parte, la prueba de esfericidad también ofreció resultados que indicaban que el análisis era pertinente (Chi-cuadrado $=5734,379$; g.l.=3000; $\mathrm{p}<0,000$ ). Además, se calculó el determinante de la matriz de correlaciones, cuyo valor fue prácticamente o $(\mathrm{D}=5.41 \mathrm{E}-10)$.

La administración del instrumento se realizó por parte de los autores durante el curso académico 2016. Tenía un carácter anónimo y fue complementado por los sujetos participantes en presencia del profesorado. Con anterioridad a la toma de los datos, se obtuvo tanto el consentimiento libre e informado del estudiantado, profesorado, así como la autorización de las autoridades del centro educativo. Para satisfacer el objetivo del estudio se procedió al desarrollo de distintos análisis, empleando el paquete estadístico SPSS. Entre ellos, se aplicó el análisis de la consistencia interna del cuestionario, el análisis de componentes principales, un estudio descriptivo de los datos, así como, una prueba t-student y análisis de varianza con prueba HSD-Tukey. 


\section{Resultados}

\section{Factores actitudinales hacia las matemáticas del estudiantado de Educación Secundaria rural}

El factor ansiedad está compuesto de nueve ítems que están referidos al sentimiento de ansiedad, temor que el estudiante manifiesta ante la materia de matemáticas, inseguridad de ser capaz de resolver situaciones que reflejen problemas matemáticos. Es necesario señalar que según Flores y Auzmendi (2015), para la interpretación del factor ansiedad, se tomará en cuenta que para los ítems que reflejen actitud negativa, entre más alta sea la puntuación de la media, menor será la actitud negativa reflejada en el estudio. Como se puede apreciar, las puntuaciones medias entre los ítems se encuentran en un intervalo de 3,15 a 4,53, siendo el ítem 17 el de menor puntuación y, el ítem 2 el de mayor puntuación. La media obtenida en el ítem 2, manifiesta que la asignatura de matemáticas no se le da mal al estudiantado del Instituto "Rubén Darío", lo que puede entenderse como un menor grado de ansiedad hacia el trato con la materia desarrollada en la disciplina de matemáticas. En el ítem 3 se aprecia que el trabajo con las matemáticas no les asusta en lo absoluto, lo que puede entenderse como una situación gratificante para el estudiantado.

Tabla 1: Medias en el factor ansiedad hacia las matemáticas

\begin{tabular}{cllcc}
\hline $\mathbf{N}^{0}$ & \multicolumn{1}{c}{ Ítems } & $\overline{\mathrm{x}}$ & $\sigma$ & $\sigma^{2}$ \\
\hline 2 & La asignatura de matemática se me da bastante mal. & 4,5333 & 0,92913 & 0,863 \\
3 & Estudiar 0 trabajar con las matemáticas no me asusta en absoluto. & 3,9333 & 0,86095 & 0,741 \\
7 & Las matemáticas es una de las asignaturas que más temo. & 3,9167 & 1,22532 & 1,501 \\
8 & Tengo confianza en mí mismo cuando me enfrento a un problema de matemáticas. & 4,0333 & 1,00788 & 1,016 \\
12 & Cuando me enfrento a un problema de matemáticas me siento incapaz de pensar con claridad. & 3,5500 & 1,09583 & 1,201 \\
13 & Estoy calmado/a y tranquilo/a cuando me enfrento a un problema de matemáticas. & 3,4833 & 1,25538 & 1,576 \\
17 & Trabajar con las matemáticas hace que me sienta nervioso/a & 3,1500 & 1,24635 & 1,553 \\
18 & No me altero cuando tengo que trabajar en problemas de matemáticas. & 3,5500 & 1,22716 & 1,506 \\
22 & Las matemáticas hacen que me sienta incomodo/a y nervioso/a. & 3,8167 & 1,14228 & 1,305 \\
\hline
\end{tabular}

Además, se evidencia claramente la seguridad y confianza que manifiesta el estudiantado tras enfrentarse a situaciones matemáticas, está claro en el ítem 7 que la asignatura no causa mucho temor según la puntuación obtenida en la media y, esto se verifica en los ítems 13 y 18 donde el estudiantado manifiesta estar calmado, tranquilo, confiando en sus capacidades matemáticas tal y como se refleja en la puntuación obtenida en el ítem 8. En el ítem 12, según la puntuación obtenida en la media y, según la interpretación dada, de acuerdo a Flores y Auzmendi (2015), el estudiantado manifiesta que, al enfrentarse a un problema de matemática, son capaces de pensar con claridad en el proceso que los conducirá a la solución de dicho problema, lo que deja ver que la ansiedad hacia las matemáticas existe en menor índice en el estudiantado y que en su mayoría se refleja una actitud positiva, frente al trato con las matemáticas.

Tabla 2: Medias en el factor agrado hacia las matemáticas

\begin{tabular}{llrrr}
\hline No & \multicolumn{1}{c}{ Ítems } & $\bar{x}$ & $\sigma$ & $\sigma^{2}$ \\
\hline 4 & Utilizar las matemáticas es una diversión. & 2,2667 & 1,92984 & 3,724 \\
9 & Me divierte el hablar con otros de matemáticas. & 3,9000 & 0,89632 & 0,803 \\
14 & Las matemáticas son agradables y estimulantes para mí. & 4,2500 & 0,96770 & 0,936 \\
24 & Si tuviera oportunidad me inscribiría en más cursos de matemáticas de los que son obligatorios. & 3,8333 & 1,13745 & 1,294 \\
\hline
\end{tabular}


El factor agrado está compuesto de 4 ítems, que reflejan el gusto o el placer que ocasiona en el estudiantado la experiencia de trabajar con las matemáticas. De acuerdo con los resultados obtenidos, las puntuaciones medias en los ítems están entre el intervalo de 2,26 a 4,25, siendo la mayor puntuación para el ítem 14 y la menor puntuación para el ítem 4. En el ítem 4 se aprecia que el estudiantado se aproxima a una posición neutral con respecto a decir si el utilizar las matemáticas es una diversión, lo que aún no nos deja ver, qué tanta satisfacción causa en el estudiantado trabajar con las matemáticas.

En el ítem 9 se manifiesta que el estudiantado está de acuerdo que le divierte hablar con otros de matemática; en este caso vemos cómo cambia la predisposición del estudiantado, lo que nos lleva a pensar que existe una manifestación de satisfacción el hecho de hablar con otros sobre matemáticas. En el ítem 14, la puntuación de la media refleja un sentimiento de placer y aprecio, al manifestar que las matemáticas les resultan agradables y estimulantes, valoración que puede entenderse como una actitud positiva hacia las matemáticas, más allá de su aprendizaje como se refleja en el ítem 24, que deja claro el interés del estudiantado, expresando estar de acuerdo de inscribirse en otros círculos de estudios de matemáticas ajenos a los obligatorios, se acentúa el componente afectivo o agrado que muestran los estudiantes hacia las matemáticas.

Tabla 3: Medias en el factor utilidad hacia las matemáticas

\begin{tabular}{lllcc}
\hline$N^{0}$ & \multicolumn{1}{c}{ Ítems } & $\bar{x}$ & $\sigma$ & $\sigma^{2}$ \\
\hline 1 & Considero las matemáticas como una materia muy necesario en mis estudios. & 4,8167 & 0,43146 & 0,186 \\
6 & Quiero llegar a tener un conocimiento más profundo de las matemáticas. & 4,4167 & 1,07816 & 1,162 \\
15 & Espero tener que utilizar poco las matemáticas en mi vida profesional. & 3,5000 & 1,49008 & 2,220 \\
16 & Considero que existen otras asignaturas más importantes que las matemáticas para mi futura profesión. & 3,3333 & 1,21665 & 1,480 \\
19 & Me gustaría tener una ocupación en la cual tuviera que utilizar las matemáticas. & 3,6833 & 1,12734 & 1,271 \\
21 & Para mi futuro profesional la matemática es una de las asignaturas más importantes que tengo que estudiar. & 4,2500 &, 98506 & 0,970 \\
\hline
\end{tabular}

La utilidad entendida como el valor que el individuo le otorga a las matemáticas es un factor que está compuesto de 6 ítems. De acuerdo con los resultados obtenidos el análisis mostró valores entre 3,33 y 4,81 para las puntuaciones medias correspondientes a los ítems que componen este factor. El ítem 1, obtuvo una media de 4,8; lo cual significa que el estudiantado valora fuertemente de acuerdo esta proposición, es decir que se puede observar que las matemáticas son valoradas por la posibilidad que da para resolver problemas cotidianos, lo que se hace más evidente en los ítems 19 y 21, donde se manifiesta total acuerdo que la matemática es una de las asignaturas más importantes a estudiar para el futuro profesional y la posibilidad de aplicarla en otras ramas del conocimiento y poder ser usada como lenguaje en cualquier ocupación, por su belleza, su potencialidad y su simplicidad (Martínez, 2008). A este análisis también se suma la puntuación media obtenida en el ítem 6, donde el estudiantado manifiesta estar de acuerdo en querer llegar a tener un conocimiento más profundo de las matemáticas. Por consiguiente, se observa que se trata de una actitud positiva percibida por el estudiantado, en cuanto a la utilidad que tienen las matemáticas en su desarrollo cognitivo y en su desempeño como futuros profesionales.

Es claro entonces que hay una responsabilidad para desarrollar y afirmar las actitudes positivas de los estudiantes y también para beneficiar la predisposición favorable hacia las matemáticas con intervenciones para mejorar la calidad de los aprendizajes, favoreciendo el desarrollo de razonamientos, argumentaciones, y en general, de procesos matemáticos fundamentados en actividades que logren estos desarrollos. 
Tabla 4: Medias en el factor motivación hacia las matemáticas

\begin{tabular}{llrrr}
\hline$N^{0}$ & \multicolumn{1}{c}{ Ítems } & $\bar{x}$ & $\sigma$ & $\sigma^{2}$ \\
\hline 5 & La matemática es demasiado teórica para que pueda servirme de algo. & 4,3000 & 1,77840 & 3,163 \\
10 & $\begin{array}{l}\text { Las matemáticas pueden ser útiles para el que decida realizar una carrera de "ciencias", } \\
\text { pero no para el resto de los estudiantes. }\end{array}$ & 3,4167 & 1,52150 & 2,315 \\
25 & La materia que se imparte en las clases de matemáticas es muy poco interesante. & 4,2000 & 1,16153 & 1,349 \\
\hline
\end{tabular}

La motivación que es entendida como el impulso del sujeto a actuar de manera determinada (Santrock, 2010), está compuesta de 3 ítems que, al ser analizados, se aprecia que las puntuaciones de sus medias están en el intervalo de 3,4 a 4,3 lo que significa que los estudiantes manifiestan una motivación intrínseca muy baja hacia el estudio de las matemáticas. En el ítem 5 los estudiantes manifiestan estar de acuerdo con que las matemáticas son demasiado teóricas para que puedan servirle de algo, lo cual deja claro que los estudiantes se muestran desmotivados para trabajar con el área de las matemáticas, y que hay una fuerza interior que provoca que se comporten de esta forma, que les impida encaminarse hacia una meta. Es evidente que el estudiantado carece de motivación intrínseca, lo que los hace ver la asignatura del todo, disfuncional en la vida cotidiana, carente de herramientas necesarias para despertar en ellos el interés y la atención por los valores contenidos en la materia.

En el ítem 25 los estudiantes manifiestan estar de acuerdo que la materia que se imparte en las matemáticas es muy poco interesante, lo que nos hace pensar que paralelo a esta respuesta existe baja motivación extrínseca. Según las puntuaciones obtenidas en las medias de este ítem se puede apreciar que los contenidos de matemáticas no resultan de mucho interés para el estudiantado, por lo cual no se evidencia en ellos el interés de aprenderla, el gusto de estudiarla y la satisfacción de cumplir con los deberes que exige el aprendizaje y tratamiento de esta disciplina.

Tabla 5: Medias en el factor confianza hacia las matemáticas

\begin{tabular}{clccc}
\hline No & \multicolumn{1}{c}{ Ítems } & $\bar{x}$ & $\sigma$ & $\sigma^{2}$ \\
\hline 11 & Tener buenos conocimientos de matemáticas incrementará mis posibilidades de trabajo. & 4,4833 & 0,70089 & 0,491 \\
20 & Me provoca una gran satisfacción el llegar a resolver problemas de matemáticas. & 4,3167 & 0,83345 & 0,695 \\
23 & Si me lo propusiera creo que llegaría a dominar bien las matemáticas. & 4,4500 & 0,72311 & 0,523 \\
\hline
\end{tabular}

El factor confianza que hace referencia a la seguridad o esperanza firme que una persona tiene de otra, de un objeto o de sí misma ante una determinada situación, está compuesto por 3 ítems en donde las puntuaciones de las medias están entre 4,31 y 4,48, por tanto, se aprecia que se trata de una actitud positiva relacionada con la capacidad de actuación. En el ítem 11 se puede apreciar que el estudiantado está de acuerdo con que tener buenos conocimientos de matemáticas incrementará sus posibilidades de trabajo; de acuerdo a la puntuación de la media en este ítem, los estudiantes valoran el aspecto cognitivo matemático, como una herramienta para garantizar un encuentro laboral o bien así la permanencia en el mismo (Conejeros, Rojas, \& Segure, 2010).

En el ítem 20 se aprecia que el estudiantado expresa estar de acuerdo que el resolver problemas de matemáticas, les causa una gran satisfacción, lo que consecuentemente da manifestaciones de tranquilidad, saber que han llegado a la solución de un determinado problema de matemática. Esto parece manifestarse como un sentimiento que emerge cuando se controla el problema, sin prisas, sin nervios y, cuando se trabaja con sosiego, serenidad, paciencia. En el ítem 23, se pone de manifiesto la confianza que tiene el estudiantado en sus capacidades matemáticas, ya que de acuerdo a la media obtenida se interpreta que están de acuerdo que si se propusieran llegarían a tener un mayor dominio de las matemáticas, lo que hace ver que existe una actitud positiva de confianza en los estudiantes hacia las matemáticas y que evidentemente se manifiesta la creencia en sus capacidades y en las sensaciones que pueden darse al momento de ser capaces de darle solución a un problema de matemáticas. 


\section{Género y grado de estudio son determinantes en las actitudes hacia las matemáticas del estudiantado de educación rural}

Con el objetivo de reforzar al lector la visión de los resultados obtenidos se presenta la figura 1, las puntuaciones medias alcanzadas por cada factor en función de la variable género, indicando los valores promedios alcanzados en la puntuación media de cada factor.



Figura 1: Medias de los factores en función de la variable género

Se realizó un análisis de las puntuaciones medias de los factores en función de la variable género. La prueba t-student para muestras independientes confirman que en los factores y la actitud global ansiedad ( $p=0,164)$; agrado ( $p=0,237)$; utilidad $(p=0,892)$; motivación $(p=0,187)$; confianza $(p=0,136)$; actitud global ( $p=0,409$ )- hombres y mujeres poseen las mismas actitudes hacia las matemáticas, ya que los valores de significancia $p$ es mayor que 0,05 .

También, se trató de estudiar si existen diferencias significativas entre especialidad en relación con las actitudes hacia las matemáticas (actitud global), el análisis de varianza de un factor muestra que $(p=0,103)$ teniendo en cuenta que $(p>0,05)$, se puede decir, que no existen diferencias estadísticamente significativas, concluyendo que el estudiantado de los grados séptimo, octavo, noveno, décimo y undécimo tienen el mismo nivel de actitud hacia las matemáticas. No obstante, la prueba post hoc (HSD-Tukey) manifiesta que el estudiantado de los grados de octavo y noveno poseen una mayor actitud hacia las matemáticas en comparación con el estudiantado de séptimo, décimo y undécimo.

Tabla 6: Comparaciones múltiples Post hoc HSD-Tukey

\begin{tabular}{|c|c|c|c|c|c|c|}
\hline \multirow{2}{*}{ (I) Grado } & \multirow{2}{*}{ (J) Grado } & \multirow{2}{*}{ Diferencia de medias (I-J) } & \multirow{2}{*}{$\begin{array}{c}\text { Error } \\
\text { estándar }\end{array}$} & \multirow{2}{*}{ Sig. } & \multicolumn{2}{|c|}{$\begin{array}{c}95 \% \text { de intervalo de } \\
\text { confianza }\end{array}$} \\
\hline & & & & & $\begin{array}{l}\text { Límite } \\
\text { inferior }\end{array}$ & $\begin{array}{l}\text { Límite } \\
\text { superior }\end{array}$ \\
\hline \multirow{4}{*}{ Séptimo } & Octavo & -4.200 & 2.853 & .585 & -12.25 & 3.85 \\
\hline & Noveno & -4.917 & 2.699 & .372 & -12.53 & 2.70 \\
\hline & Décimo & -.500 & 3.069 & 1.000 & -9.16 & 8.16 \\
\hline & Undécimo & 2.000 & 2.638 & .941 & -5.44 & 9.44 \\
\hline
\end{tabular}




\begin{tabular}{|c|c|c|c|c|c|c|}
\hline \multirow{2}{*}{ (I) Grado } & \multirow{2}{*}{ (J) Grado } & \multirow{2}{*}{ Diferencia de medias (I-J) } & \multirow{2}{*}{$\begin{array}{l}\text { Error están- } \\
\quad \text { dar }\end{array}$} & \multirow{2}{*}{ Sig. } & \multicolumn{2}{|c|}{$\begin{array}{l}95 \% \text { de intervalo de } \\
\text { confianza }\end{array}$} \\
\hline & & & & & $\begin{array}{l}\text { Límite } \\
\text { inferior }\end{array}$ & $\begin{array}{l}\text { Límite } \\
\text { superior }\end{array}$ \\
\hline \multirow{4}{*}{ Octavo } & Séptimo & 4.200 & 2.853 & .585 & -3.85 & 12.25 \\
\hline & Noveno & -.717 & 3.065 & .999 & -9.36 & 7.93 \\
\hline & Décimo & 3.700 & 3.396 & .811 & -5.88 & 13.28 \\
\hline & Undécimo & 6.200 & 3.011 & .253 & -2.29 & 14.69 \\
\hline \multirow{4}{*}{ Noveno } & Séptimo & 4.917 & 2.699 & .372 & -2.70 & 12.53 \\
\hline & Octavo & .717 & 3.065 & .999 & -7.93 & 9.36 \\
\hline & Décimo & 4.417 & 3.267 & .660 & -4.80 & 13.63 \\
\hline & Undécimo & 6.917 & 2.866 & .127 & -1.17 & 15.00 \\
\hline \multirow{4}{*}{ Décimo } & Séptimo & .500 & 3.069 & 1.000 & -8.16 & 9.16 \\
\hline & Octavo & -3.700 & 3.396 & .811 & -13.28 & 5.88 \\
\hline & Noveno & -4.417 & 3.267 & .660 & -13.63 & 4.80 \\
\hline & Undécimo & 2.500 & 3.217 & .936 & -6.57 & 11.57 \\
\hline \multirow{4}{*}{ Undécimo } & Séptimo & -2.000 & 2.638 & .941 & -9.44 & 5.44 \\
\hline & Octavo & -6.200 & 3.011 & .253 & -14.69 & 2.29 \\
\hline & Noveno & -6.917 & 2.866 & .127 & -15.00 & 1.17 \\
\hline & Décimo & -2.500 & 3.217 & .936 & -11.57 & 6.57 \\
\hline
\end{tabular}

\section{Conclusiones}

El objetivo central de esta investigación, fue analizar las actitudes hacia las matemáticas del estudiantado de Educación Secundaria procedente de una escuela rural, encontrando una actitud globalmente favorable de 82,32. Esto se debe al modelo de formación de la educación rural que se sustenta en la creación, diseño e implementación de ambientes de aprendizajes donde se aplican los conceptos y propiedades de la aritmética, estadística, álgebra, geometría y trigonometría en situaciones del entorno rural y vida cotidiana del estudiantado. Los resultados de la investigación, también señalan que los factores actitudinales se manifiestan al momento de resolver situaciones problemas (Flores \& Auzmendi, 2016) tipificándose de la manera siguiente:

En el factor ansiedad, se concluye que el estudiantado al enfrentarse a un problema de matemática es capaz de pensar con claridad en el proceso que los conducirá a la solución de dicho problema. El factor agrado, se expresa en términos de inscribirse en otros círculos de estudios de matemáticas ajenos a los obligatorios, se acentúa el componente afectivo o agrado que muestran los estudiantes hacia las matemáticas. En el factor utilidad, el estudiantado manifiesta que las matemáticas son útiles, porque dan la posibilidad de resolver problemas cotidianos, así como importantes para el futuro profesional y la posibilidad de aplicarla en otras ramas del conocimiento y poder ser usada como lenguaje en cualquier ocupación, por su belleza, su potencialidad y su simplicidad.

En el factor motivación que es entendido como el impulso del sujeto a actuar de manera determinada (Santrock, 2010), los estudiantes muestran una motivación intrínseca muy baja hacia el estudio de las matemáticas. Esto se debe, porque, el estudiantado puede apreciar que los contenidos de matemáticas no resultan de mucho interés para el estudiantado, por lo cual no se evidencia en ellos el interés de aprenderla, el gusto de estudiarla y la satisfacción de cumplir con los deberes que exige el aprendizaje y tratamiento de esta disciplina. 
El factor confianza que hace referencia a la seguridad o esperanza firme que una persona tiene de otra, de un objeto o de sí misma ante una determinada situación. El estudiantado tiene confianza en sus capacidades matemáticas, porque al tener un mayor dominio de las matemáticas, hace ver que existe una actitud positiva de confianza en los estudiantes hacia las matemáticas y que evidentemente se manifiesta la creencia en sus capacidades $y$ en las sensaciones que pueden darse al momento de ser capaces de darle solución a un problema de matemáticas.

Con referencia a las variables género y grado de estudio. Se señala que, hombres y mujeres tienen el mismo grado de actitud hacia las matemáticas. Esto se debe porque la educación rural se desarrolla habilidades y capacidades para incentivar y orientar el trabajo colectivo, la responsabilidad social compartida, la cooperación y la solidaridad, lo que contribuye a la formación de hombres y mujeres solidarios y participativos, que respetan y valoran la diversidad de género. También, las actitudes hacia las matemáticas se manifiestan como elemento relevante en la variable grado de estudio. Se puede decir, que no existen diferencias estadísticamente significativas, concluyendo que el estudiantado de los grados séptimo, octavo, noveno, décimo y undécimo tiene el mismo nivel de actitud hacia las matemáticas. Sin embargo, el estudiantado de los grados de octavo y noveno poseen una mayor actitud hacia las matemáticas en comparación con el estudiantado de séptimo, décimo y undécimo.

Finalmente, se concluye que las actitudes hacia las matemáticas son parte de la formación del estudiantado de Educación Secundaria rural, ya que les permite desarrollar habilidades y destrezas para conocer, valorar, comprender y manejar las emociones y sentimientos de forma armónica en la resolución de situaciones matemáticas vinculadas con la vida cotidiana del estudiantado. Además, que la formación de las actitudes hacia las matemáticas ayuda a promover la equidad y la igualdad de oportunidades, basados en la comunicación, la justicia, el respeto, la estima, la tolerancia, la solidaridad, la cooperación y el intercambio de experiencias entre hombres y mujeres, encaminados a nuevos pensamientos, comportamientos y estilo de vida que se trasmite a las distintas generaciones.

\section{Bibliografía}

Albert, M. (2006). La investigación educativa. Claves teóricas. Madrid: MCGraw-Hill.

Auzmendi, E. (1992). Las actitudes hacia la matemática-estadistica en la enseñanza media y universitaria. Bilbao: Mensajero.

Bisquerra, R. (2012). Metodología de la investigación educativa. Madrid: Muralla.

Calvo, A., \& Susinos, T. (2010). Prácticas de investigación que escuchan la voz del alumnado: Mejorar la universidad indagando la experiencia. Profesorado, Revista de Currículum y Formación de Profesorado, 14(3), 75-88.

Conejeros, M. L., Rojas, J., \& Segure, T. (2010). Confianza: Un valor necesario y ausente en la educación chilena. Mexico: Perfiles Educativos.

Flores, W. O., \& Auzmendi, E. (2016). Los problemas de comprensión del álgebra en estudiantes universitarios. Ciencia e Interculturalidad, 19(2), 54-64. DOI: http://dx.doi.org/10.5377/ rci.v19i2.3119

Flores, W., O. \& Auzmendi, E. (2015). Análisis de la estructura factorial de una escala de actitud hacia las matemáticas. Aula de Encuentro, 17(1), 45-77.

Gil, J., Rodríguez, G., \& García, E. (1995). Estadística básica aplicada a las Ciencias de la Educación. Sevilla: Kronos.

Gil, N., Guerrero, E., \& Blanco, L. (2006). El dominio afectivo en el aprendizaje de las matemáticas. Revista Educación Matemática Psicoeducativa, 4(1), 47-72.

Gómez-Chacón, I. (2000). Matemática emocional. Los afectos en el aprendizaje matemático. Madrid: Narcea.

Hernández, R., Fernández, C., \& Baptista, M. (2010). Metodología de la investigación. México: McGraw-Hill.

Hidalgo, S., Maroto, A., \& Palacios, A. (2005). El perfil emocional matemático como predictor de rechazo escolar: Relación con las destrezas y los conocimientos desde una perspectiva evolutiva. Revista de Educación Matemática, 17(2), 89-116. 
Lim, L., Tso, T., \& Lin, F. (2009). Assessing science students' attitudes to mathematics: a case study on a modeling proyect with mathematical software. International Journal of mathematical Education in Science and Technolgy, 40(4), 441-453.

Martínez, O. (2008). Actitud hacia la matemática. Sapiens: Revista Universitaria de Investigación, 237-256.

NCTM. (200o). Principles and standards for school mathematics. VA: NCTM.
Sabariego, M. (2004). El proceso de investigación. En R. Bisquerra, Metodología de la investigación educativa (págs. 127-163). Madrid: La Muralla.

Santrock, J. (2010). Psicología de la Educación. México: McGraw-Hill.

Schofield, H. (1982). Sex, Grade Level, and the Relationship between Mathematics Attitude and Achievement in Children. Journal of Educational Research, 7, 280-284. 